\title{
Korrespondenzen.
}

\section{Zur Prioritätsfrage in der Tuberculinherstellung und} -anwendung.

Von J. Den ys, Professor an der Universität in Löwen(Belgíen).

In No. 51 (vom 21. Dezember 1905) dieser Wochenschrift beansprucht C. Spengler die Priorität in der Anwendung der filtrierten, uneingeengten Tuberkelbacillenbouillon bei der Behandlung der menschlichen Tuberculose. Den Anlaß zu Spenglers Ausführungen gab mein Buch: Le bouillon filtré du bacille de la tuberculose dans le traitement de la tuberculose humaine, in dem ich eine Behandlung der Tuberculose darstelle vermittels einer Tuberkelbacillenbouillon, die, um sie von allen Keimen zu befreien, einfach durch eine Porzellankerze filtriert und gebraucht wird, ohne vorher der Hitze oder chemischen Reagenzien ausgesetzt zu sein. Dr. Spengler bemerkt, daß er dieses Präparat zuerst angewandt hat, und führt aus, daß Koch vor 1893 Versuche mit demselben bei Tieren gemacht und im selben Jahre Spengler beauftragt hat, die Versuche am Meerschweinchen und 
Menschen fortzusetzen. Spengler veröffentlichte seine Resultate im Jahre 1897.

Es geht zunächst aus Spenglers eigenem Artikel hervor, daB Koch seine Experimente nicht veröffentlicht hat; man kann mir also auch nicht vorwerfen, eine Idee des großen Gelehrten aufgenommen zu haben, um so weniger, als $\mathrm{K}$ och niemals den Gebrauch der filtrierten Bouillon beim Menschen empfohlen hat. Kochs erstes Tuberculin, jetzt altes Kochsches Tuberculin, unterscheidet sich sehr von der einfach filtrierten Bouillon, weil es erstens der Einwirkung hoher Temperaturen $\left(70^{\circ}\right)$ ausgesetzt wird und reicher an Bacillenkörperderivaten ist. Ferner hat $\mathrm{Koch}$ sein erstes Tuberculin aufgegeben, um es gegen TR und später gegen seine Bacillenemulsion zu vertauschen, die er für das wirksamste Mittel hält. TR jedoch und die Bacillenemulsion, die ausschlieBlich $m i t$ dem Körper der Bacillen hergestellt werden, sind von der filtrierten Bouillon vollkommen verschieden.

Was Spenglers Arbeiten anbetrifft, so wurden sie, wie gesagt, erst 1897 veröffentlicht. Ich selbst habe mich jedoch schon seit 1896 mit der Wirkung der filtrierten Bouillon bei der menschlichen und experimentellen Tuberculose eingehend befaßt, wie man aus den Berichten des Pariser Tuberculosekongresses von 1897 ersehen kann. Meine Untersuchungen haben also unabhängig von denjenigen Spenglers begonnen, und es scheint mir, daß Spengler um so weniger Anlaß zu Reklamationen hat, als er selbst die filtrierte Bouillon der menschlichen Tuberculose, an der ich festhalte, gegen diejenige der Ti ertuberculose vertauscht hat, der jch aus $G$ ründen, die ich in meinem Buche dargelegt habe, keinerlei spezielle Eigentümlichkeiten zuschreiben kann. Es sollte auch nicht vergessen werden, daß $\mathbf{S}$ pengle $\mathbf{r}$ bis zum Jahre 1897 die filtrierte Bouillon nur bei etwa 50 Kranken angewandt hatte und daB er erst wieder begonnen hat, über diese Substanz zu veröffentlichen, nachdem mein Tuberculin angefangen hatte, seit 1902 in Davos mit Erfolg verwandt zu werden.

Dagegen habe ich in der ganzen Zwischenzeit trotz mannigfacher Anfeindungen keinen Augenblick aufgehört, für die filtrierte Bouillon einzutreten, soda $B$ entweder von mir selbst oder von anderen mehr als 2000 Kranke, die ich persönlich untersucht habe, behandelt worden sind. In keinem Lande hat ferner die Behandlung mit einem Tuberculin soviel Anhänger gefunden wie in Belgien.

Und schließlich verbietet noch ein anderer Grund, den ich für ausschlaggebend ansehe, mich einfach als Nachahmer Kochs oder Spenglers hinzustellen: Wie aus meinem Buche hervorgeht, besteht zwischen der Art und Weise, wie Spengle r seine Bouillon anwendet, und der meinigen ein absoluter Untersschied. Spengler hält nämlich Reaktionen (sogar $39^{\circ}$ ) für sehr wünschenswert, da er sie für die Heilung als unumgänglich nötig betrachtet. Ich dagegen halte die Reaktionen, und seien sie auch noch so leicht, für schädlich; höchstens dürfen sie in Ausnahmefällen geduldet werden und auch dann nur schwach angedeutet sein. Deswegen beginne ich mit $0,000001 \mathrm{~g}, 0,0000001 \mathrm{~g}$ und sogar mit 0,000 000 $01 \mathrm{~g}$. und aus diesem Grunde habe ich in meinem Buche eine ganze Reihe von zum größten Teil neuen Verhaltungsmaßregeln gegeben, die mir eine zehnjährige Erfahrung diktiert hat und deren Einhaltung bei der Anwendung des Heilmittels von kapitaler Wichtigkeit ist. Diese Regeln gestatten, die fieberhaften Tuberculosen ohne jegliche Gefahr in Angriff zu nehmen. Der Vorwurf, Koch und Spengler kopiert zu haben, ist also nicht verdient, und zwar: 1. weil meine Untersuchungen ein Jahr vor der ersten Veröffentlichung von Spenglers Resultaten begonnen haben und Spengler selbst zeitweise auf weitere Versuche verzichtet hat; 2 . weil $K o c h$ in seinen Schriften nie die filtrierte Bouillon als Heilmethode beim Menschen angeraten hat, wohl aber sein altes Tuberculin, das vom meinigen tief verschieden ist, und später seine Tuberkel- und seine Bacillenemulsion; 3. weil meine Anwendung essentiell von der. jenigen Kochs und Spenglers verschieden ist. Gerade die Anwendung spielt aber meines Erachtens eine höchst wichtige Rolle bei der Erzielung des Erfolges. 